\title{
NOTE ON IRREGULAR PRIMES
}

L. CARLITZ

1. We recall that a prime $p$ is irregular if it divides the numerator of at least one of the numbers

$$
B_{2}, B_{4}, \cdots, B_{p-3},
$$

where $B_{m}$ denotes a Bernoulli number in the even-suffix notation. Jensen has proved that there exist infinitely many irregular primes of the form $4 n+3$ (for the proof see [3, p. 82]; see also [4]).

In this note we give a simple proof of the weaker result that the number of irregular primes is infinite. We also prove a like result corresponding to the prime divisors of the Euler numbers.

The letter $p$ will always denote a prime $>2$.

2. We shall make use of the following well known properties of Bernoulli numbers. For proofs see [2, Chaps. 13, 14].

$$
\begin{array}{rlrl}
B_{m} & \equiv 0\left(\bmod p^{r}\right) & \left(p^{r} \mid m, p-1 \nmid m\right) . \\
p B_{m} & \equiv-1(\bmod p) & & (p-1 \mid m) . \\
\frac{B_{m+r(p-1)}}{m+r(p-1)} & \equiv \frac{B_{m}}{m}(\bmod p) & & (p-1 \nmid m) .
\end{array}
$$

(2.2) is contained in the Staudt-Clausen theorem, while (2.3) is a special case of Kummer's congruence for the Bernoulli numbers. Note that both members of $(2.3)$ are integral $(\bmod p)$.

A prime divisor of the numerator of $B_{m} / m$ may be called a proper divisor of $B_{m}$; this is not quite the terminology of [4].

It follows from (2.3) that if $p$ is a proper divisor of $B_{m}$ then it is also a divisor of $B_{s}$, where

$$
m \equiv s(\bmod p-1) \quad(0<s<p-1) ;
$$

that $s \neq 0$ is a consequence of (2.2). Thus a proper divisor of any $B_{m}$ is certainly irregular. Now assume that there are only a finite number of irregular primes $p_{1}, \cdots, p_{k}$, and consider the number $B_{M}$, where

$$
M=2 t \prod_{i=1}^{k}\left(p_{i}-1\right)
$$

If we put

Received by the editors August 23, 1953. 


$$
B_{M} / M=N_{M} / D_{M}
$$$$
\left(\left(N_{M}, D_{M}\right)=1\right) \text {, }
$$

it follows from the above and (2.2) that $N_{M}= \pm 1$. For, as already remarked, a prime divisor of $N_{M}$ is a proper divisor of $B_{M}$ and therefore irregular; but by (2.2) and (2.4) the irregular primes $p_{1}, \cdots, p_{k}$ occur in the denominator of $B_{M}$. On the other hand it is clear from

$$
\frac{B_{2 m}}{2 m}=(-1)^{m-1} \frac{2(2 m-1) !}{(2 \pi)^{2 m}} \sum_{r=1}^{\infty} \frac{1}{r^{2 m}}
$$

that $\left|B_{2 m} / 2 m\right| \rightarrow \infty$ as $m \rightarrow \infty$. Since $t$ in (2.4) is at our disposal, it is evident that this contradicts $\left|N_{M}\right|=1$.

3. Some criteria in terms of Euler numbers for the first case of Fermat's last theorem have been given. Vandiver [5] has proved that if

$$
x^{p}+y^{p}=z^{p}
$$

is satisfied, then

$$
E_{p-3} \equiv 0(\bmod p)
$$

Gut [1] has proved that if

$$
x^{2 p}+y^{2 p}=z^{2 p}
$$

is satisfied, then

$$
E_{p-3} \equiv E_{p-5} \equiv E_{p-7} \equiv E_{p-9} \equiv E_{p-11} \equiv 0(\bmod p) .
$$

Here the $E_{m}$ denote Euler numbers in the even suffix notation.

We accordingly define a prime $p$ as irregular with respect to the Euler numbers if it divides at least one of the numbers

$$
E_{2}, E_{4}, \cdots, E_{p-3} \text {. }
$$

We shall prove that the number of such primes is infinite.

Analogous to (2.3) we now have [2, Chap. 14]

$$
E_{m+r(p-1)} \equiv E_{m}(\bmod p)
$$$$
(m \geqq 1) \text {. }
$$

We have also the property [2, p. 273]: if $p-1 \mid m$,

$$
E_{m} \equiv \begin{cases}0(\bmod p) & (p \equiv 1(\bmod 4)) \\ 2(\bmod p) & (p \equiv 3(\bmod 4)) .\end{cases}
$$

We shall say that $p$ is a proper divisor of $E_{m}$ provided $p \mid E_{m}$ and $p-1 \nmid m$; clearly in view of (3.5) only primes of the form $4 n+1$ can be improper divisors. 
It follows from (3.4) that if $p$ is a proper divisor of $E_{m}$ then it is also a divisor of $E_{s}$, where

$$
m \equiv s(\bmod p-1) \quad(0<s<p-1) .
$$

Let us now assume that there are only a finite number of irregular primes (relative to the Euler numbers) $p_{1}, \cdots, p_{k}$, and consider the number $E_{M}$, where

$$
M=4 t \Pi\left(p_{i}-1\right)+2 .
$$

By (3.4)

$$
E_{M} \equiv E_{2} \equiv-1\left(\bmod p_{i}\right) \quad(i=1, \cdots, k)
$$

Thus

$$
\left(E_{M}, p_{1} p_{2} \cdots p_{k}\right)=1
$$

also since $M \equiv 2(\bmod 4)$, it is clear that $E_{M}$ has no improper divisors. Consequently $E_{M}= \pm 1$. But since

$$
E_{2 m}=(-1)^{m} \frac{4(2 m) ! 2^{2 m}}{\pi^{2 m+1}} \sum_{r=0}^{\infty} \frac{(-1)^{r}}{r^{2 m+1}}
$$

it is evident that $\left|E_{M}\right| \rightarrow \infty$.

\section{REFERENCES}

1. M. Gut, Eulersche Zahlen und grosser Fermat'scher Satz, Comment. Math. Helv. vol. 24 (1950) pp. 73-99.

2. N. Nielsen, Traité élémentaire des nombres de Bernoulli, Paris, 1923.

3. H. S. Vandiver and G. E. Wahlin, Algebraic numbers II, Bulletin of the National Research Council, no. 62, 1928.

4. H. S. Vandiver, Note on the divisors of the numerators of Bernoulli's numbers, Proc. Nat. Acad. Sci. U. S. A. vol. 18 (1932) pp. 594-597.

5. - Note on Euler number criteria for the first case of Fermat's last theorem, Amer. J. Math. vol. 62 (1940) pp. 79-82.

Duke UnIVERSITY 